Rev. Elev. Méd. vét. Pays trop., 1974, 27 (1) : 109-114

\title{
Extériorisation des potentialités du zébu Gobra. Résultats des abattages pratiqués en 1972
}

\author{
par J. P. DENIS (*), J. VALENZA $\left(^{*}\right)$ et A. I. THIONGANE $\left(^{* *}\right)$ \\ (avec la collaboration technique de A. DOUCOURE (**)
}

\section{RESUME}

Ces nouveaux abattages confirment les résultats obtenus précédemment. Les animaux de 18 mois ont donné des carcasses de 234, 229, $166 \mathrm{~kg}$ pour les non castrés, 195 et 170 pour les castrés avec des rendements respectifs de $62,2,60,7-60,8$ et $57,5-59,2$ p. 100 . En ce qui concerne les mâles de 4 ans et demi, les poids de carcasse sont de 394 et $368 \mathrm{~kg}$, avec des rendements de 63,7 et 62,2 p. 100 . Quant à la femelle abattue en même temps à 4 ans 3 mois, sa carcasse pèse $270 \mathrm{~kg}$ avec un rendement de 61,6 p. 100 .

\section{INTRODUCTION}

L'expérience d'extériorisation des potentialités génétiques du zébu Gobra, démarrée en 1968 , s'est poursuivie en 1972. Le présent document rapporte les résultats des abattages effectués au cours de l'année 1972.

\section{MATERIEL ET METHODES}

\section{Animaux}

\subsection{Expérimentaux}

Les abattages ont été effectués en deux temps :

- En mai 1972, 5 jeunes animaux, dont 2 castrés, âgés en moyenne de 18 mois, produits des femelles d'extériorisation dont, par conséquent, les conditions générales d'environnement ont été assez largement améliorées;

- En novembre 1972, 3 animaux du lot 1968 d'extériorisation: deux mâles et une

$\left(^{*}\right)$ I.E.M.V.T., Laboratoire national de l'Elevage, B.P. 2057, Dakar-Hann (Sénégal).

(**) Centre de Recherches zootechniques de Dahra, Sénégal. femelle. Cette femelle, depuis sa mise à la reproduction (janvier 1970) n'a donné aucun produit dans un lot où l'âge du premier vêlage a été de $933 \pm 45$ jours, soit : 31 mois.

\subsection{Témoins}

Le lot témoin est toujours constitué par les animaux nés au C.R.Z. de Dahra au cours des années 1965-1966 et 1967.

\section{Alimentation}

Les animaux ont continué à recevoir, en plus du pâturage naturel, un concentré distribué à volonté. La composition de ce concentré est restée la même depuis que les animaux ont atteint leur seconde année:

- Maïs . . . . . . . 10

— Son de blé . . . . . . . . 40

- Son de maïs . . . . . . 17

— Son de sorgho . . . . . . 29,50

— Tourteau d'arachide . . . . 0,50

- Carbonate de calcium . . . 2,25

- Chlorure de sodium . . . . 0,50

-. Complément vitaminé . . . 0,25 
Le concentré titre 0,85 U.F. et $100 \mathrm{~g}$ de M.A.D. par kg.

Il faut noter que le pâturage mis à la disposition des animaux est très pauvre, à la suite de saisons des pluies catastrophiques $(210 \mathrm{~mm}$ en 1970, $276 \mathrm{~mm}$ en 1971, $273 \mathrm{~mm}$ en 1972 avec une période de sécheresse intercalaire de 53 jours).

\section{Observations}

Les observations classiques ont été effectuées à savoir: pesées et mensurations mensuelles régulières.

\section{RESULTATS}

\section{Animaux abattus en mai 1972}

\subsection{Croissance pondérale}

Les 5 jeunes animaux abattus, nés en 1970, sont sacrifiés à 18 mois en moyenne; il s'agit de contrôler en particulier les rendements, associés à la recherche d'une coloration correcte de la viande qui, chez les animaux abattus en 1971 à 11 mois, avait été jugée trop claire pour du baby beef et trop rouge pour répondre aux critères de coloration du veau.

Deux des animaux ont été castrés deux mois avant l'abattage, leur croissance sera individualisée. Le tableau $n^{\circ}$ I permet de connaître les caractéristiques pondérales des animaux abattus.

Donc le gain moyen quotidien est de $0,555 \mathrm{~g}$ chez les animaux castrés (2992 et 4020$)$ et de $0,600 \mathrm{~g}$ chez les animaux entiers.

Les évolutions pondérales de ces animaux comparées à celles des animaux témoins et d'extériorisation nés en 1968 apparaissent au tableau II.

\subsection{Abattages}

Les résultats des abattages apparaissent au tableau $\mathrm{n}^{n}$ III.

\section{COMMENTAIRES :}

1.2.1. Le pourcentage de perte au jeûne est important sur ces jeunes animaux. Mais ce jeûne a duré $45 \mathrm{~h}$, avec un voyage de $270 \mathrm{~km}$ en bétaillère qui, par accident, s'est déroulé aux heures les plus chaudes de la journée avec une hygrométrie très faible.

1.2.2. La perte au ressuyage est pratiquement inexistante au bout de 24 heures.

1.2.3. Les rendements sont excellents, mais inférieurs chez les animaux castrés $(2992$ - 4020) 58,4 p. 100 contre 61,2 p. 100.

1.2.4. Le poids de contenu de panse reste relativement faible.

1.2.5. L'indice de gras est peu élevé $(1 / 4$ moyenne).

1.2.6. La couleur de la viande est jugée bonne mais cependant pas tout à fait assez foncée pour du baby beef.

\subsection{Découpe de demi-gros détaillée}

En 1971, une découpe anatomique complète d'une demi-carcasse avait été effectuée afin de contrôler en particulier les proportions respectives de muscles, os et gras.

En mai 1972, c'est une découpe de demigros qui a été effectuée sur 2 demi-carcasses de mâles (4020) et (4031) et la demi-carcasse de femelles (3740) selon la technique dite «parisienne $»$.

TABLEAU $\mathrm{N}^{\circ} \mathrm{I}$

\begin{tabular}{|c|c|c|c|c|c|c|c|}
\hline & $\begin{array}{c}\text { Date à la } \\
\text { naissance }\end{array}$ & $\begin{array}{c}\text { Poids à la } \\
\text { naissance }\end{array}$ & $\begin{array}{c}\text { Poids à } \\
\text { I'abattage }\end{array}$ & $\begin{array}{c}\text { Gain de } \\
\text { poids }\end{array}$ & $\begin{array}{c}\text { Age en } \\
\text { jours }\end{array}$ & $\begin{array}{c}\text { Gain moyen } \\
\text { quotidien }\end{array}$ & $\begin{array}{c}\text { Age en } \\
\text { mois }\end{array}$ \\
\hline $\begin{array}{c}2992 \\
\text { castrê) } \\
4010\end{array}$ & 9.9 .70 & 31 & 370 & 339 & 606 & 0,560 & 20 \\
4011 & 25.10 .70 & 31 & 407 & 376 & 560 & 0,670 & 19 \\
4021 & 25.10 .70 & 25 & 402 & 377 & 560 & 0,675 & 19 \\
(castré) & 19.11 .70 & 23 & 315 & 292 & 535 & 0,545 & 18 \\
4031 & $28,1.71$ & 25 & 308 & 283 & 465 & 0,610 & 16 \\
\hline
\end{tabular}


TABLEAU $N^{\circ}$ II

\begin{tabular}{|c|c|c|c|c|c|c|c|c|c|c|c|c|}
\hline & \multicolumn{3}{|c|}{ Naissance } & \multicolumn{3}{|c|}{6 mois } & \multicolumn{3}{|c|}{12 mois } & \multicolumn{3}{|c|}{18 mois } \\
\hline & $\mathrm{N}$ & $\bar{x}$ & \pm & $\mathrm{N}$ & $\bar{x}$ & \pm & $\mathrm{N}$ & $\bar{x}$ & \pm & $\mathrm{N}$ & $\bar{x}$ & \pm \\
\hline Témoins & 227 & 25,0 & 0,5 & 162 & 96,2 & 2,7 & 199 & 144,0 & 20 & 145 & 196,6 & 6,1 \\
\hline Extêriorisés 68 & 14 & 21,3 & 1,6 & 15 & 126,3 & 12,2 & 14 & 248,7 & $1 B, 1$ & 14 & 381,2 & 18,1 \\
\hline Extériorisés 70 & 9 & 26,4 & 2,50 & 9 & 166,5 & 28,6 & 6 & 255,7 & 40,7 & 4 & 354 & 94,4 \\
\hline
\end{tabular}

TABLEAU $N^{\circ}$ III

\begin{tabular}{|c|c|c|c|c|c|c|}
\hline \multirow{2}{*}{\multicolumn{2}{|c|}{ Poids avant jeûne }} & 2992 & 4010 & 4011 & 4021 & 4031 \\
\hline & & 370 & 407 & 402 & 315 & 308 \\
\hline \multicolumn{2}{|c|}{ Poids après jeûne } & 339 & 376 & 377 & 287 & 273 \\
\hline \multicolumn{2}{|c|}{ p.100 perte jeûne } & 8,37 & 7,62 & 6,22 & 8,89 & 11,4 \\
\hline \multicolumn{2}{|c|}{ Poids carcasse froide } & 195 & 23 & 229 & 169 & 166 \\
\hline \multicolumn{2}{|c|}{ Poids carcasse chaude } & 195 & 234 & 229 & 179 & 166 \\
\hline \multicolumn{2}{|c|}{ p. 100 perte ressuyage $(24 \mathrm{~h})$} & - & 0,4 & - & 0,6 & - \\
\hline \multicolumn{2}{|c|}{ Renjement } & 57,5 & 62,2 & 60,7 & 59,2 & 60,8 \\
\hline \multicolumn{2}{|c|}{ Rendement vrai } & 63,2 & 65,8 & 64,9 & 63,3 & 64,5 \\
\hline \multicolumn{2}{|c|}{ p.100 contenu panse } & 15,6 & 8,8 & 9,8 & 10,9 & 9,5 \\
\hline \multicolumn{2}{|c|}{ p.100 5e quartier } & 26,8 & 24,3 & $24, B$ & 24,9 & 24,0 \\
\hline \multirow{7}{*}{ p. 100} & Epaule & 21,3 & 19,66 & 22,38 & - & - \\
\hline & Pis & 11,5 & 12,0 & 11,0 & - & - \\
\hline & Panneau & 4,6 & 5,8 & 5,8 & - & - \\
\hline & Train de côtes & 9,4 & 8,0 & 9,7 & - & - \\
\hline & Globe & 46,3 & 45,8 & 44,5 & - & - \\
\hline & Bosse & 2,0 & 1,7 & 1,83 & - & - \\
\hline & Gras de rognon & 1,0 & 1,8 & 1,5 & - & - \\
\hline \multicolumn{2}{|c|}{ Cuir } & 31,0 & 32,0 & 33,4 & 25,0 & 24,3 \\
\hline \multicolumn{2}{|c|}{ Queue } & 1,0 & 1,3 & 1,3 & - & - \\
\hline \multicolumn{2}{|c|}{ Longueur carcasse } & 113,5 & 114,5 & 115,5 & 111,5 & 107,5 \\
\hline \multicolumn{2}{|c|}{ Epaisseur de 1a cuisse } & 22,7 & 23,8 & 23,6 & 23,7 & 21,5 \\
\hline \multicolumn{2}{|c|}{ Epaisseur plat de côtes } & 3,1 & 3,4 & 3,4 & 3,6 & 3,4 \\
\hline \multicolumn{2}{|c|}{ Indice de compacitê } & 1,31 & 2,05 & 1,98 & 1,52 & 1,54 \\
\hline \multicolumn{2}{|c|}{ Conformation } & 4,5 & 5 & 4,5 & $4+$ & $4+$ \\
\hline
\end{tabular}

Rectification : Epaule : Lire 21,6; 19,6; 22,3. 
Pour chacune des demi-carcasses, apparaît le pourcentage représenté par le morceau rapporté au poids total de la demi-carcasse (tableau $\mathrm{n}^{\circ}$ IV).

On note que les proportions d'avant et d'arrière sont respectivement de 60 et 40 p. 100 .

La découpe détaillée a été ensuite effectuée, les résultats apparaissent au tableau $n^{*} \mathrm{~V}$.

\section{Animaux abattus en novembre 1972}

\subsection{Croissance pondérale}

Les animaux abattus sont nés en 1968. Ils appartiennent au lot d'extériorisation mâle pour les $\mathrm{n}^{\circ} 2721$ et 2731 , au lot femelle pour le $n^{0} 3740$ qui est stérile.

Le tableau $\mathrm{n}^{\circ}$ VI montre Ies caractéristiques de ces animaux sur le plan de la croissance.

On constate que la vitesse de croissance a été fortement diminuée durant cette dernière période, le poids obtenu constituant approximativement le maximum que l'on puisse atteindre avec ces animaux Gobra du C.R.Z. élevés dans les conditions expérimentales de l'extériorisation.

Par rapport aux abattages précédents, les résultats sont comparables. II faut signaler cependant que le pourcentage de gras est moins élevé sur ces dernières carcasses et qu'en particulier sur celles des deux mâles, la graisse de couverture est tout juste suffisante.

La perte de poids au ressuyage est toujours faible.

Les rendements sont excellents même chez la femelle ( 62,5 p. 100 en moyenne). La couleur de la viande est normale.

\subsection{Résultats des abattages}

Ils sont présentés au tableau $\mathrm{n}^{\circ}$ VII.

\subsection{Découpe de demi-gros}

Seule la demi-carcasse gauche de la femelle 3740 a été découpée et on peut lire les résultats sur le tableau $\mathrm{n}^{\mathrm{o}} \mathrm{V}$.

\section{Animaux témoins}

Pour les découpes de demi-gros, 2 demicarcasses d'animaux courants prises à l'abattoir de Dakar ont été étudiées dans les mêmes conditions que les animaux d'extériorisation.
TABLEAU N ${ }^{\circ}$ IV

\begin{tabular}{|c|c|c|}
\hline & 4020 & 4031 \\
\cline { 2 - 3 } & $\mathrm{p} .100$ & $\mathrm{p} .100$ \\
\hline Epaule & 26,64 & 28,69 \\
\hline Cuisse & 26,0 & 24,50 \\
\hline Train de côtes & 10,13 & 7,14 \\
\hline Aloyau & 13,43 & 12,80 \\
\hline Panneau & 5,12 & 4,92 \\
\hline Pis & 10,74 & 12,56 \\
\hline
\end{tabular}

TABLEAU $\mathrm{N}^{\circ} \mathrm{V}$

Pourcentages des différents morceaux de demi-gros par rapport au poids de la demi-carcasse gauche froide

\begin{tabular}{|c|c|c|c|}
\hline & 4021 & $4 \quad 031$ & 3740 \\
\hline Epaule (paleron et collier) & 26,6 & 28,7 & 24,6 \\
\hline Collier & 7,9 & 8,4 & 4,4 \\
\hline Paleron & 18,7 & 20,4 & 20,2 \\
\hline Gîte de devant & 3,1 & 2,7 & 2,4 \\
\hline Griffe & $\overline{1,4}$ & 1,3 & $\overline{1, \overline{6}}$ \\
\hline Derrière de paleron & $\overline{0,8}$ & 1,7 & 3,4 \\
\hline Charolaise & 1,4 & 1,3 & 1,4 \\
\hline Jumeaux Pot at feu & 0,4 & 0,5 & 0,5 \\
\hline Bumeaux Beefteak & 1,0 & 1,1 & 0,9 \\
\hline Macreuse + os & 9,9 & 11,3 & $\bar{X}$ \\
\hline Désossêe & 8,0 & 9,5 & 8,0 \\
\hline Dessus & 2,4 & 2,6 & 1,5 \\
\hline Dessous & $3, \overline{5}$ & 4,6 & $3, \bar{B}$ \\
\hline Boule & 2,1 & 2,2 & 2,7 \\
\hline Piêce parée & $\mathrm{X}$ & 1,4 & $\overline{\mathrm{X}}$ \\
\hline Cuisse & 26,0 & 24,5 & $2.3,1$ \\
\hline Gîte (coupe d'êtê) & 3,9 & 3,3 & 2,9 \\
\hline Globe & 21,8 & 21,0 & \\
\hline Tende de tranche & 6,2 & $6, \overline{3}$ & 5,6 \\
\hline Seneile & $7, \overline{1}$ & 6,7 & 6,9 \\
\hline Tranche grasse & 4,6 & 4,3 & 3,7 \\
\hline Hanche & 4,7 & 4,3 & 4,4 \\
\hline Aiguillette de rumsteak & 0,8 & 0,9 & 1,5 \\
\hline Rums teak & 3,3 & 3,3 & 2,3 \\
\hline Araignée & $\overline{\mathrm{x}}$ & $\mathrm{x}$ & 0,1 \\
\hline Train de côtes (11 côtes) & 10,1 & 7,1 & 8,3 \\
\hline Echine & 1,9 & 1,2 & 1,5 \\
\hline Aloyau + aiguillette barone & 13,4 & 12,8 & 6,8 \\
\hline Faux-filet & 3,8 & 5,3 & 3,8 \\
\hline Aiguillette barone & $\overline{0}, \bar{B}$ & 0,8 & 0,8 \\
\hline Côtes dẻcouvertes & 4,5 & 1,5 & 2,9 \\
\hline Côtes couvertes & 3,4 & 3,4 & 3,6 \\
\hline Filet mignon & 0,2 & 0,2 & 0,1 \\
\hline Panneau & $5, \overline{1}$ & 4,9 & 9,8 \\
\hline Plat de côtes & 3,3 & 3,5 & $\bar{x}$ \\
\hline Bavette aloyau & 1,8 & 1,3 & $\mathrm{X}$ \\
\hline Pis & 10,7 & 12,5 & 13,4 \\
\hline Flanchet & 4,8 & 7,3 & $\overline{\mathrm{X}}$ \\
\hline Poitrine & 5,9 & 5,2 & $\mathrm{X}$ \\
\hline Filet & 2,2 & 2,2 & 2,2 \\
\hline Queve & 1,0 & 1,1 & 1,4 \\
\hline Bosse & 2,2 & 2,2 & 2,5 \\
\hline Rognon & 0,4 & 0,5 & 0,4 \\
\hline Gras de rognon & 0,8 & 1,6 & 3,1 \\
\hline Graisse de bassin & 0,6 & 0,8 & 2,8 \\
\hline Graisse inguí & 0,8 & 0,8 & $\begin{array}{l}\text { 2, B ré- } \\
\text { tromam- } \\
\text { maire }\end{array}$ \\
\hline
\end{tabular}


TABLEAU $N^{\circ} \mathrm{VI}$

\begin{tabular}{|c|c|c|c|c|c|c|}
\hline & $\begin{array}{c}\text { Date de } \\
\text { naissance }\end{array}$ & $\begin{array}{c}\text { Poids à 1a } \\
\text { naissance }\end{array}$ & Age à l'abattage & $\begin{array}{c}\text { Poids abattage } \\
\text { avant jeûne }\end{array}$ & $\begin{array}{c}\text { Gain de } \\
\text { poids }\end{array}$ & $\begin{array}{c}\text { Gain moyen } \\
\text { quotidien }\end{array}$ \\
\hline 2721 & 29.05 .68 & 24 & $1731 \mathrm{j} \mathrm{4}$ ans $6 \mathrm{~m}$ & 620 & 596 & 0,344 \\
\hline 2731 & 19.06 .68 & 24 & $1613 \mathrm{j} 4$ ans $5 \mathrm{~m}$. & 639 & 615 & 0,381 \\
\hline 3740 & 23.08 .68 & 22 & $1540 \mathrm{j} 4$ ans 3 m. & 457 & 435 & 0,282 \\
\hline
\end{tabular}

TABLEAU $\mathrm{N}^{\circ} \mathrm{VII}$

\begin{tabular}{|c|c|c|c|}
\hline & $2 \overline{721}$ & 2731 & 3740 \\
\hline Poids ayant jeûne & $6 \overline{20}$ & 639 & 457 \\
\hline Poids après jeâne & 592 & 619 & 438 \\
\hline p.100 perte jeûne & 4,5 & 3,1 & 4,2 \\
\hline Poids carcasse chaude & 368 & 394 & 270 \\
\hline Poids carcasse froide & 357 & 389 & 267 \\
\hline p.100 perte ressuyage & 3,0 & 1,3 & 1,1 \\
\hline Rendement & 62,2 & 63,7 & 61,6 \\
\hline Rendement vrai & 65,3 & 67,2 & 67,4 \\
\hline p. 100 contenu panse & 5,5 & 5,3 & 8,7 \\
\hline p. 100 e quartier & 22,4 & 25,7 & 26,7 \\
\hline $\begin{array}{l}\text { En pourcentage du poids } \\
\text { de la carcasse froide (G) } \\
\text { Pis }\end{array}$ & 15,1 & 15,8 & 13,6 \\
\hline Epaule & 24,1 & 25,1 & 21,8 \\
\hline Panneau & 5,2 & 4,9 & 6,5 \\
\hline Train de côtes & $B, 3$ & 6,9 & 6,9 \\
\hline Globe & 44,6 & 43,7 & 48,4 \\
\hline Bosse & 2,8 & 2,3 & 1,3 \\
\hline Gras de rognon & 0,9 & 1,4 & 2,1 \\
\hline Cuir & $\overline{47}$ & $4 \overline{4}$ & 29,5 \\
\hline Queue & 1,6 & 1,6 & 1,7 \\
\hline Longueur carcasse & 131,7 & 135,0 & 125,7 \\
\hline Epaisseur cuisse & 28,8 & 30,5 & 26,6 \\
\hline Epaisseur plat de cótes & 50,0 & 49,5 & 38,5 \\
\hline Indice de compacité & 2,5 & 2,7 & 2,0 \\
\hline Conformation carcasse & 4,5 & 4,5 & 4 \\
\hline
\end{tabular}

Enfin, celles de deux animaux issus d'une expérience d'embouche effectuée à Sangalkam également ont été découpées.

Ces différents résultats apparaissent au tableau $\mathbf{n}^{\circ}$ VIII (Pourcentage des différents morceaux par rapport à la demi-carcasse poids).

Pour apprécier l'une des carcasses, la côte correspondant à la $12^{\mathrm{e}}$ vertèbre dorsale a été prélevée pour déterminer les proportions respectives d'os, de muscle et de graisse. Les résultats sont les suivants :

$$
\begin{aligned}
& \text { - os . . . . . . 17,4 p. } 100 \\
& \text { - muscle . . . . . } 56,7 \text { p. } 100 \\
& \text { — graisse . . . . . } 25,8 \text { p. } 100
\end{aligned}
$$

De même la surface du longissimus dorsi a été appréciée par planimétrie au niveau de la section passant entre la $11^{\mathrm{e}}$ et la $12^{\mathrm{e}}$ vertèbre dorsale. Cette surface est de :

- $53 \mathrm{~cm}^{2}$ chez les témoins;
TABLEAU $N^{\circ}$ VIII

\begin{tabular}{|l|c|c|c|c|}
\hline & \multicolumn{2}{|c|}{ Témoins } & \multicolumn{2}{c|}{ Embouche } \\
\hline Epaule & 23,0 & 28,8 & 24,6 & 24,5 \\
\hline Cuisse & 38,9 & 33,8 & 25,1 & 23,9 \\
\hline Train de côtes & 16,3 & 7,7 & 8,1 & 8,3 \\
\hline Panneau & 17,7 & 10,2 & 9,6 & 10,8 \\
\hline Pis & 10,9 & 10,0 & 12,7 & 12,2 \\
\hline $\begin{array}{l}\text { Aloyau + aiguillette } \\
\text { barone }\end{array}$ & 5,8 & 3,7 & 6,1 & 6,2 \\
\hline Filet & 1,8 & 2,0 & 2,2 & 2,3 \\
\hline
\end{tabular}

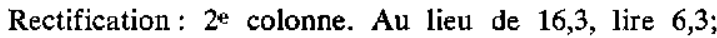
au lieu de 17,7 , lire 7,7 .

- $82 \mathrm{~cm}^{2}$ chez les femelles - 3740 ;

- $65 \mathrm{~cm}^{2}$ chez le mâle de 18 mois.

\section{CONCLUSIONS}

Ces abattages d'animaux d'extériorisation clôturent l'opération sur le plan de l'étude bouchère. En effet, la croissance des animaux semble arrivée à son maximum aux alentours de 42 mois et, si l'étude de l'indice de consommation n'a pu être effectuée systématiquement, il apparaît cependant que cet indice est devenu progressivement irraisonnablement élevé et donc anti-économique.

Les performances relevées sont toujours aussi bonnes sur les plans du poids total de viande obtenu et du rendement. Il faut souligner que l'indice de gras, fort chez les animaux plus jeunes ( 36 mois), est devenu plus faible chez les derniers animaux abattus, la graisse de couverture dans un cas ayant même été jugée insuffisante. 


\section{Remerciements}

Nous tenons particulièrement à remercier, Messieurs les professeurs P. CUQ et J. ROZIER de l'Ecole Inter-Etats des Sciences et Médecine vétérinaires de Dakar pour le précieux concours qu'ils nous ont apporté dans les opérations de découpe de demi-gros détaillées.

\section{SUMMARY}

\section{Phenotypic potentialities of Gobra zebu cattle (Senegal)}

These new slaughterings confirm the results obtained before. The animals of 18 months old produced carcasses of 234, 229, $166 \mathrm{~kg}$ for non castrated ones, 195 and $170 \mathrm{~kg}$ for castrated with respective dressing percentages of $62,2,60,7-60,8$ and $57,5-59,2$ per 100 .

Concerning males of 4 and a half years old, the carcass weights are 394 and $368 \mathrm{~kg}$ with dressing percentages of 63,7 and 62,2 per 100 .

As to the female of 4 years and 3 months old, slaughtered in the some time the carcass weights $270 \mathrm{~kg}$ with a dressing percentage of 61,6 per 100 .

\section{RESUMEN}

Exteriorización de los potenciales del cebú Gobra. Resultados de las matanzas efectuadas en 1972 (Senegal)

Estas nuevas matanzas confirman los resultados anteriormente obtenidos. Los animales de 18 meses de edad no castrados daron canales de $234,229,166 \mathrm{~kg}$, los castrados: de 195 y 170 con rendimientos respectivamente de $62,2,60,7-60,8$ y $57,5-59,2$ p. 100 .

En lo concerniente a los machos de 4 años y media de edad, los pesos de canales son de 394 y $368 \mathrm{~kg}$, con rendimientos de 63,7 y 62,2 p. 100 .

En cuanto a la hembra matada al mismo tempo a 4 años y 3 meses de edad, su canal pesa $270 \mathrm{~kg}$ con un rendimiento de $61,6 \mathrm{p}$. 100. 